Original Article

Journal of Epilepsy Research pISSN 2233-6249 / elSSN 2233-6257

Received November 23, 2017 Accepted June 5, 2018

Corresponding author: Kwang Ki Kim, MD, PhD Department of Neurology, Dongguk University Ilsan Hospital, 27 Dongguk-ro, Ilsandong-gu, Goyang 10326, Korea

Tel. +82-31-961-7200

Fax. +82-31-961-3277

E-mail; neukim@duih.org

\title{
Seizure-Related Cortical Volume Alterations in Alzheimer's Disease: A Preliminary Study
}

\author{
Jarang Hahm, PhD', Kwang Ki Kim, MD, PhD', Dong Wook Kim, MD, PhD ${ }^{2}$ \\ ${ }^{1}$ Department of Neurology, Dongguk University Ilsan Hospital, Goyang; ${ }^{2}$ Department of Neurology, Konkuk \\ University School of Medicine, Seoul, Korea
}

Background and Purpose: Alzheimer's disease (AD) leads to cognitive dysfunction and neuronal loss, both of which can be exacerbated by seizures. For the treatment and diagnosis of $A D$, it is imperative to identify the cortical characteristics of comorbidities of $A D$ such as seizures. The present study investigated the alterations in cortical volumes in patients with comorbid $A D$ and seizures.

Methods: In this retrospective study, magnetic resonance T1-weighted brain images were collected from six patients with early $A D$ or amnestic mild cognitive impairment without seizures (AD-No Seizure, age: $66.17 \pm 4.92$ years) and six individuals with seizures (AD-Seizure, age: $80.33 \pm 4.63$ years). The gray matter volumes estimated from the T1 images were compared between the groups using nuisance variables (e.g., age). Subsequently, a correlation analysis was performed to investigate the relationship between cortical structure and global cognitive function.

Results: AD-Seizure group showed volumetric alterations compared with AD-No Seizure group. In the volumetrically altered regions, correlation analysis revealed that the AD-Seizure group showed a positive correlation between the mini-mental state examination (MMSE) score and cortical volume, with smaller volumes than the AD-No Seizure group in the right parahippocampal gyrus, left angular gyrus, and middle temporal gyrus. The AD-No Seizure group showed negative correlations with MMSE score in the volume of right inferior frontal gyrus and cerebellar culmen and a positive correlation with the volume of the left middle frontal gyrus.

Conclusions: Our findings revealed that smaller temporal region volumes are predictive of cognitive dysfunction in $A D$ patients with seizures. Given that these temporal areas overlap with regions showing abnormal brain activities in $A D$ patients with seizures, these results suggest synergistic effects of $A D$ and seizures on cortical volume and cognitive function. (2018;8:33-40)

Key words: Alzheimer's disease, Seizures, Gray Matter

\section{Introduction}

Alzheimer's disease (AD) is the most common cause of dementia. Patients with AD present with memory impairment and cognitive dysfunction, and report difficulty in activities of daily living. Cognitive dysfunction in AD can progress more rapidly, ${ }^{1,2}$ and occur earlier ${ }^{3}$ in patients with comorbid seizures than in those without. The incidence of epilepsy in AD patients is three times greater than in age-matched controls. ${ }^{4}$ This result was found not only in the late, ${ }^{5}$ but also in the early stage of $A D$, and in mild cognitive impairment $(M C l){ }^{3}$ In light of the harmful consequences relating to cognitive function and increased risk of epilepsy in AD patients, treatment and diagnosis of
AD with comorbid epilepsy is imperative, and the examination of its basic neural properties is essential.

The increased risk of epilepsy in AD patients suggests a possible pathological link between epilepsy and AD. A high level of beta-amyloid $(A B)$ in the brain can cause aberrant excessive neuronal activ$i t y,{ }^{6,7}$ which in turn facilitates the synaptic release of $A \beta$, leading to cell death and cognitive decline in the AD brain. ${ }^{8}$ This vicious synergistic effect has been seen in pathological alterations at the cellular-level of the postmortem human, ${ }^{9}$ and transgenic mouse brain. ${ }^{10}$

In this study, we investigated alterations in cortical volume of $A D$ patients with seizures, compared to those without seizures. As a preliminary study, we compared cortical volumes between six early $A D$ 
or MCl patients with seizures, and six without seizures using voxel-based morphometry analysis. We hypothesized that AD patients with seizures would have smaller cortical volume than those without seizures, especially in seizure-related areas. These findings would provide regional-level evidence and lend support to previous cellular-level findings.

\section{Methods}

\section{Subjects}

We designed a retrospective study by collecting brain magnetic resonance images (MRIs) of patients who visited Dongguk University Ilsan Hospital from January to May of 2017. We included patients who were diagnosed with amnestic $\mathrm{MCl}$ or probable $\mathrm{AD}$ according to the National Institute of Neurological and Communicative Disorders and Stroke and the Alzheimer's Disease and Related Disorders Association (NINCDS-ADRDA) criteria. ${ }^{11}$ The inclusion criteria were a diagnosis of probable $A D$, a global deterioration scale (GDS) of 3 or 4, a score between 10-26 on the Korean version of the mini-mental state examination (MMSE), age over 50 years, and an absence of a history of other psychiatric disorders. Of the patients who satisfied the above criteria, we grouped those patients without clinical seizures as "AD patients without seizures (AD-No Seizure)", and those with clinical seizures as "AD patients with seizures (AD-Seizure)". As it was more difficult to find $A D / M C l$ patients with seizures than those without seizures, we adjusted the number of patients in the AD-No Seizure group to match that of the AD-Seizure group while matching as closely as possible for age and gender. In total, we included 12 patients: six patients in the AD-No Seizure and six patients in the AD-Seizure group. The demographic information and neuropsychological results of the selected patients are summarized in Table 1.

Individual brain structure and activity were examined with MRI, electroencephalography (EEG) recording, and medical examination. In the AD-No Seizure group, the patients' EEG recordings showed normal electrical brain activity. In the AD-Seizure group, all patients had episodes of dialeptic seizures (i.e., an alteration of consciousness) ${ }^{12}$ without generalized tonic-clonic seizures. One patient had a vertiginous aura, and one patient experienced visual aura. Although definite epileptic discharges were observed in only one patient, semi-rhythmic theta activity across frontotemporal areas was observed in all patients in the AD-Seizure group. With anti-epileptic drug treatment, all patients with seizures had no, or reduced, recurrence of dialeptic seizure or amnesic events. Examination of all MRIs revealed that white matter hyperintensity of all patients was minimal.

\section{Brain MRI acquisition}

For each participant, a high-resolution T1-weighted 3-dimensional anatomical scan was obtained using 3T Magnetom Skyra MRI scanner (Siemens, Germany) at the Dongguk University Ilsan Hospital, Goyang, Korea. The 3-dimensional magnetization-prepared rapid gradient-echo sequence protocol was used with the following parameters: repetition time $=2,300 \mathrm{~ms}$; echo time $=2.26 \mathrm{~ms}$; field of view $=256 \times 256 \mathrm{~mm}$; flip angle $=8^{\circ} ;$ spatial resolution $=0.5 \times 0.5$ $\times 0.5 \mathrm{~mm}$. Two subjects from each group were imaged with slightly different protocols: echo time: 2.98 ; flip angle $=9^{\circ}$; field of view $=248 \times 256 \mathrm{~mm}$; spatial resolution $=1.0 \times 1.0 \times 1.2 \mathrm{~mm}$.

\section{Gray matter volume calculation}

Based on the T1-weighted MRIs of the included patients, gray matter volumes (GMV) were calculated using voxel-based morphometry analysis in the SPM12 toolbox (Wellcome Department of Imaging Neuroscience Group, London, UK) executed in Matlab (MathWorks, Inc., Natick, MA, USA). First, an individual T1-weighted image was segmented, in each participant's native space, into gray

Table 1. Demographic information and neuropsychological evaluation of the study groups

\begin{tabular}{lccc}
\hline & AD-No Seizure & AD-Seizures & $t$-value \\
\hline N (male:female) & $6(2: 4)$ & $6(2: 4)$ & NA \\
GDS (N) & Score 3 $(4)$, score 4 (2) & Score 3 (2), score 4 (4) & NA \\
Age (years) & $66.17 \pm 4.92(60-73)$ & $80.33 \pm 4.63(75-85)$ & $-5.14^{*}$ \\
Education (years) & $6.42 \pm 3.88(0.5-12)$ & $4.00 \pm 3.15(0.5-8)$ & 1.19 \\
MMSE & $22.67 \pm 1.86(20-25)$ & $19.50 \pm 4.97(12-24)$ & 1.46 \\
Depression & $4.50 \pm 3.94(1-12)$ & $4.67 \pm 4.68(1-13)$ & -0.07 \\
\hline
\end{tabular}

AD, Alzheimer's disease; AD-No Seizure, AD patients without seizures; AD-Seizure, AD patients with seizures; N, number; NA, not applicable; GDS, global deterioration scale; MMSE, mini-mental state examination (Korean version). ${ }^{*} p<0.0005$. 
matter, white matter, cerebrospinal fluid, skull, soft tissue outside of the brain, and other stuff outside of the head. Then, segmented images were spatially normalized into Montreal Neurological Institute (MNI) space using the DARTEL ${ }^{13}$ toolbox in SPM12. The default settings were maintained, and the sample-derived template with a voxel size of $1.5 \times 1.5 \times 1.5 \mathrm{~mm}$ was used. The normalized gray matter images were modulated with the Jacobian determinant maps from the anatomical standardization stage, allowing a quantitative assessment of the regional GMV. Finally, the GMV images were smoothed with an $8 \mathrm{~mm}$ full-width-at-half-maximum isotropic Gaussian kernel. As a nuisance variable for the subsequent group analysis, the individual total intracranial volume was calculated as the sum of the gray matter, white matter, and cerebrospinal fluid (CSF) in each subject's native space using SPM12.

\section{Statistical analysis}

Group comparisons were performed between the AD-No Seizure and the AD-Seizure group. Age, gender, total intracranial volume, years of education, MMSE, and imaging protocol type were treated as nuisance variables in the general linear model. After applying the gray matter mask based on the sample-derived gray matter template obtained from the DARTEL procedure, the alpha level was set at $p<$ 0.001 with a cluster size $\leq 5$ without correction for multiple comparisons. The gray matter mask only included voxels with gray matter intensity $\geq 0.45$. While this threshold value was quite strict, it successfully excluded voxels showing white matter hyperintensity from the analysis. For a preliminary study with a small number of observations (i.e., subjects), uncorrected $p<0.001$ is quite conservative, so we also applied a more liberal alpha level of uncorrected $p<0.005$, to allow observation of cortical volume alterations in more regions. To label the peaks of significant clusters according to Brodmann area (BA), the peak coordinates were converted from MNI space to Talairach space, ${ }^{14}$ and anatomical region and BA were identified using Talairach Client. ${ }^{15,16}$

\section{Relationship between GMV and cognitive function}

To examine the relationship between cortical volume and cognitive function, we estimated correlation coefficients between GMV and MMSE score. The GMV values of each subject were extracted at all peak voxels that showed significant group differences at $p<0.005$, and age, gender, total intracranial volume, years of education, and imaging protocol type were regressed out. GMV residuals were used to compute Pearson's correlation coefficients, which were transformed into $z$-val- ues using Fisher's $z$ transformation, for each group separately, and group differences in correlation coefficients were tested. The absolute values of $z$-scores were compared between groups using an equation as follows: $\left(\operatorname{abs}\left(z_{1}\right)-a b s\left(z_{2}\right)\right) / \sqrt{\frac{1}{n_{1}-3}+\frac{1}{n_{2}-3}}$ where a subscript of 1 indicated the AD-No Seizure group, and a subscript of 2 indicated the AD-Seizure group. The indicated $z$-score was the number of patients, and abs was a function yielding an absolute value.

\section{Results}

\section{GMV alterations in the AD brain with and without comorbid seizures}

Group comparisons revealed that the AD-Seizure group showed smaller cortical volume than the AD-No Seizure group in the right inferior parietal lobule, the left lingual gyrus, and cerebellar region ( $p<$ 0.001 ) (Table 2). In addition, at the more liberal threshold of $p<$ 0.005 , differences were observed in the left medial frontal (BA 10), angular (BA 39), middle temporal (BA 39) gyri, cerebellar tonsil; the right superior frontal (BA 6), medial frontal (BA 9), inferior frontal (BA 45), parahippocampal (BA 35), lingual (BA 18) gyri and cerebellar tuber (Table 2).

On the contrary, larger cortical volumes were observed in the AD-Seizure than in the AD-No Seizure group in the left middle frontal (BA 8), postcentral (BA 2), and right middle temporal (BA 39) gyri ( $p$ $<0.001$ ) (Table 2). At an alpha level of 0.005 , the left middle frontal gyrus (BA 6), inferior parietal lobule (BA 40), precuneus (BA 7, 31), cuneus (BA 17, 18), and middle temporal gyrus (BA 37) also emerged. In the right hemisphere, a cluster consisting of the cingulate (BA 32) and medial frontal (BA 6) gyri; a cluster consisting of the postcentral (BA 3), precentral (BA 4), and superior frontal (BA 6) gyri; and the cerebellar declive were observed (Table 2).

\section{Relationship between GMV alterations and cognitive function}

To examine a relationship between cortical volume alterations and cognitive function, we calculated the correlation coefficient between GMV and MMSE scores separately for each group. We hypothesized that there would be a positive correlation between cortical volume and MMSE score, such that smaller cortical volumes would be associated with cognitive dysfunction in the AD-Seizure group. As expected, in the AD-Seizure group there was a positive association be- 
tween MMSE score and peak volume of the right parahippocampal region ( $r=0.88, p=0.02)$, the left middle temporal gyrus $(r=0.86$, $p=0.03)$, and the angular gyrus $(r=0.87, p=0.03)$ (Fig. 1A-C, Table 2). Interestingly, the AD-No Seizure group showed a significant negative correlation between MMSE score and peak volume of the right inferior frontal gyrus $(r=-0.86, p=0.03)$, and the left cerebellar culmen ( $r=-0.83, p=0.04$ ) (Fig. 1D and E, Table 2).
Regions with significantly greater cortical volume in the AD-Seizure group did not show any significant correlation with MMSE score in that group. However, there was a positive correlation between cortical volume and MMSE score in the left middle frontal gyrus (BA 8) in the AD-No Seizure group (Fig. 2, Table 2). Comparisons of correlation coefficients between groups were not significantly different based on $z$-statistics ( $p>0.05$ ) (Table 2).

Table 2. Alterations in gray matter volume depending on seizure presence in Alzheimer's disease (AD) patients $(P<0.005$, cluster size $\geq 5$, uncorrected for multiple comparisons) and their relationship with cognitive function, as assessed by the MMSE

\begin{tabular}{|c|c|c|c|c|c|c|c|c|c|c|c|c|}
\hline \multirow[b]{2}{*}{ Contrast } & \multirow[b]{2}{*}{$\begin{array}{l}\text { Cluster } \\
\text { size }\end{array}$} & \multirow[b]{2}{*}{$\begin{array}{l}t \text {-value of } \\
\text { peak }\end{array}$} & \multirow[b]{2}{*}{ Side } & \multirow[b]{2}{*}{ Lobe } & \multirow[b]{2}{*}{ Region } & \multirow[b]{2}{*}{ BA } & \multicolumn{3}{|c|}{$\begin{array}{c}\text { Talairach } \\
\text { coordinate }\end{array}$} & \multicolumn{3}{|c|}{ Correlation with MMSE } \\
\hline & & & & & & & $x$ & $\mathrm{y}$ & z & $\begin{array}{l}\text {-value for } \\
A D \\
\text {-No Seizures }\end{array}$ & $\begin{array}{c}r \text {-value for } \\
A D \\
\text {-Seizures }\end{array}$ & $\begin{array}{c}z \text {-value for } \\
\text { group } \\
\text { difference }\end{array}$ \\
\hline \multirow{15}{*}{$\begin{array}{l}\text { AD-No } \\
\text { Seizure > } \\
\text { AD-Seizure }\end{array}$} & 8 & 6.74 & L & Frontal & Medial frontal gyrus & 10 & -6 & 50 & 3 & 0.08 & 0.84 & -1.41 \\
\hline & 7 & 5.67 & L & Frontal & Medial frontal gyrus & 10 & -7 & 63 & 12 & 0.11 & 0.76 & -1.08 \\
\hline & 18 & 6.07 & L & Parietal & Angular gyrus & 39 & -48 & -61 & 32 & -0.2 & $0.87^{*}$ & -1.36 \\
\hline & 20 & 11.48 & L & Temporal & Middle temporal gyrus & 39 & -42 & -59 & 20 & -0.04 & $0.86^{\star}$ & -1.54 \\
\hline & 33 & 6.27 & L & Posterior & Cerebellar tonsil & NA & -13 & -44 & -39 & -0.14 & 0.40 & -0.35 \\
\hline & 183 & $14.38^{\dagger}$ & L & Anterior & Cerebellar culmen & NA & -7 & -65 & -9 & $-0.83^{*}$ & -0.30 & 1.05 \\
\hline & & $9.90^{\dagger}$ & L & Occipital & Lingual gyrus & 18 & -12 & -72 & -8 & -0.93 & -0.57 & 1.23 \\
\hline & & 6.52 & $\mathrm{R}$ & Anterior & Cerebellar culmen of vermis & NA & 3 & -66 & -11 & -0.37 & 0.28 & 0.13 \\
\hline & 19 & 7.46 & $\mathrm{R}$ & Frontal & Superior frontal gyrus & 6 & 19 & 3 & 63 & -0.18 & -0.48 & -0.42 \\
\hline & 14 & 5.89 & $\mathrm{R}$ & Frontal & Medial frontal gyrus & 9 & 6 & 46 & 18 & -0.33 & 0.63 & -0.49 \\
\hline & 5 & 5.01 & $\mathrm{R}$ & Frontal & Inferior frontal gyrus & 45 & 52 & 37 & 8 & $-0.86^{*}$ & -0.06 & 1.51 \\
\hline & 57 & $16.00^{\dagger}$ & $R$ & Parietal & Inferior parietal lobule & 40 & 49 & -46 & 49 & -0.13 & 0.63 & -0.75 \\
\hline & 6 & 4.95 & $\mathrm{R}$ & Limbic & Parahippocampal gyrus & 35 & 31 & -27 & -24 & 0.02 & $0.88^{*}$ & -1.64 \\
\hline & 9 & 5.58 & $R$ & Occipital & Lingual gyrus & 18 & 18 & -75 & -8 & -0.77 & -0.36 & 0.80 \\
\hline & 33 & 7.59 & $\mathrm{R}$ & Posterior & Cerebellar tuber & NA & 19 & -89 & -30 & -0.34 & 0.20 & 0.18 \\
\hline \multirow{18}{*}{$\begin{array}{l}\text { AD-Seizure } \\
\text { > AD-No } \\
\text { Seizure }\end{array}$} & 58 & $13.65^{\dagger}$ & $\mathrm{L}$ & Frontal & Middle frontal gyrus & 8 & -30 & 24 & 44 & 0.73 & 0.44 & 0.57 \\
\hline & 15 & 9.97 & $\mathrm{~L}$ & Frontal & Middle frontal gyrus & 6 & -43 & 2 & 47 & 0.62 & 0.25 & 0.58 \\
\hline & 14 & 8.55 & L & Frontal & Middle frontal gyrus & 8 & -27 & 15 & 45 & $0.86^{*}$ & 0.59 & 0.74 \\
\hline & 127 & $9.30^{\dagger}$ & $\mathrm{L}$ & Parietal & Postcentral gyrus & 2 & -55 & -22 & 45 & 0.42 & 0.21 & 0.29 \\
\hline & 5 & 5.82 & L & Parietal & Inferior parietal lobule & 40 & -33 & -42 & 53 & 0.72 & 0.47 & 0.50 \\
\hline & 5 & 5.65 & L & Parietal & Precuneus & 7 & -3 & -58 & 54 & 0.28 & 0.13 & 0.20 \\
\hline & 21 & 6.93 & $\mathrm{~L}$ & Parietal/occipital & Precuneus/cuneus & $31 / 17$ & -12 & -57 & 24 & 0.69 & 0.31 & 0.64 \\
\hline & 10 & 6.82 & L & Temporal & Middle temporal gyrus & 37 & -43 & -63 & -2 & 0.77 & 0.51 & 0.57 \\
\hline & 24 & 8.88 & L & Occipital & Cuneus & 18 & -9 & -70 & 28 & 0.26 & -0.04 & 0.28 \\
\hline & 29 & 6.80 & $\mathrm{R}$ & Frontal & Cingulate gyrus & 32 & 4 & 21 & 38 & 0.59 & -0.01 & 0.82 \\
\hline & & 5.41 & $\mathrm{R}$ & Frontal & Medial frontal gyrus & 6 & 9 & 17 & 43 & 0.66 & 0.25 & 0.64 \\
\hline & 8 & 5.85 & $\mathrm{R}$ & Frontal & Superior frontal gyrus & 6 & 4 & 5 & 51 & 0.24 & 0.30 & -0.09 \\
\hline & 9 & 5.47 & $\mathrm{R}$ & Frontal & Superior frontal gyrus & 6 & 6 & 23 & 55 & 0.74 & 0.09 & 1.06 \\
\hline & 127 & $8.93^{\dagger}$ & $\mathrm{R}$ & Temporal & Middle temporal gyrus & 39 & 55 & -65 & 10 & 0.76 & 0.43 & 0.65 \\
\hline & & 6.75 & $\mathrm{R}$ & Temporal & Superior temporal gyrus & 22 & 59 & -56 & 17 & 0.69 & 0.50 & 0.36 \\
\hline & 40 & 8.53 & $\mathrm{R}$ & Parietal & Postcentral gyrus & 3 & 19 & -37 & 68 & 0.69 & 0.45 & 0.46 \\
\hline & & 7.78 & $\mathrm{R}$ & Frontal & Precentral gyrus & 4 & 18 & -29 & 68 & 0.63 & 0.37 & 0.44 \\
\hline & 7 & 5.55 & $\mathrm{R}$ & Posterior & Cerebellar declive & NA & 28 & -87 & -21 & 0.5 & 0.36 & 0.21 \\
\hline
\end{tabular}

$\mathrm{BA}$ is not applicable for cerebellar regions.

MMSE, mini-mental state examination; BA, Brodmann area; AD-No Seizure, AD patients without seizures; AD-Seizure, AD patients with seizures; L, left; $R$, right; NA, not applicable.

${ }^{*} p<0.05$ for $r$-values.

${ }^{\dagger} p<0.001$ for $t$-values of peak voxels. 
A

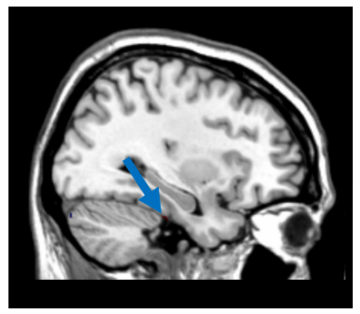

B

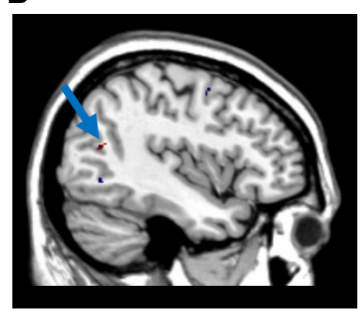

C

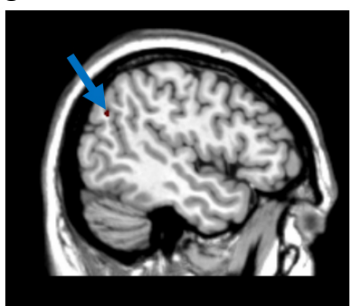

D

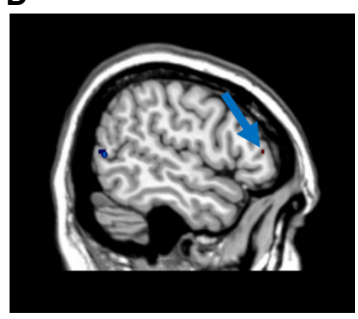

E

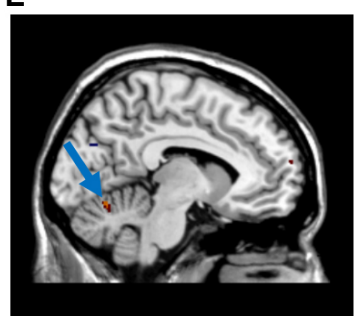

AD-No Seizure
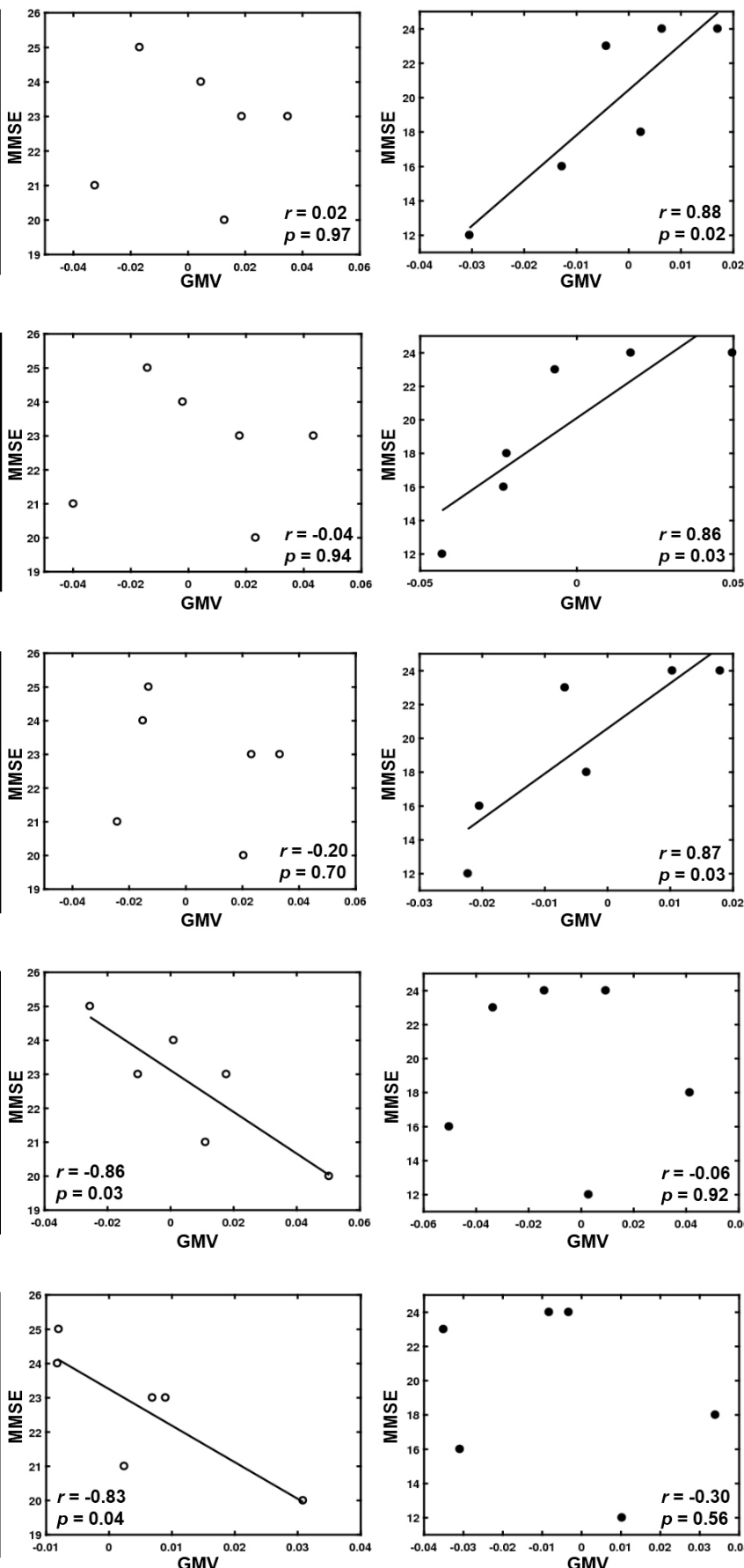

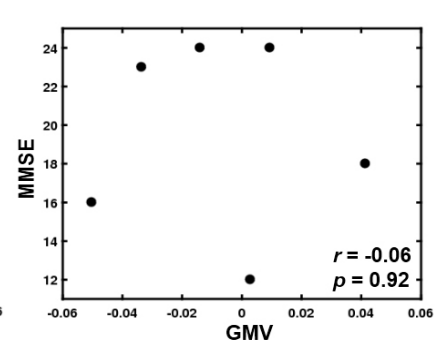

AD-Seizure
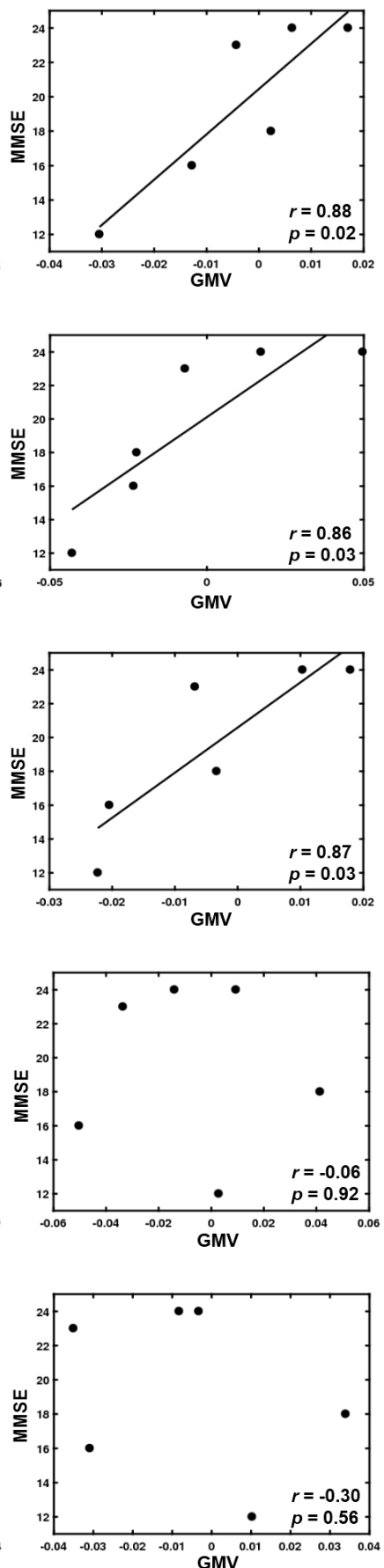

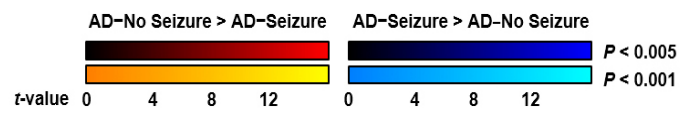

Figure 1. Correlation between MMSE score and gray matter volume of each region showing smaller volume in patients with Alzheimer's disease (AD) who experience seizures than those who did not $(P<0.001$ and $P<0.005$, cluster size $\geq 5$, uncorrected for multiple comparisons). (A) Right parahippocampal gyrus. (B) Left middle temporal gyrus. (C) Left angular gyrus. (D) Right inferior frontal gyrus. (E) Left cerebellar culmen. An arrow in each figure indicated a peak voxel served to perform the correlation analysis. MMSE, mini-mental state examination; AD-No Seizure, AD patients without seizures; $A D-S e i z u r e, A D$ patients with seizures; GMV, gray matter volume. 


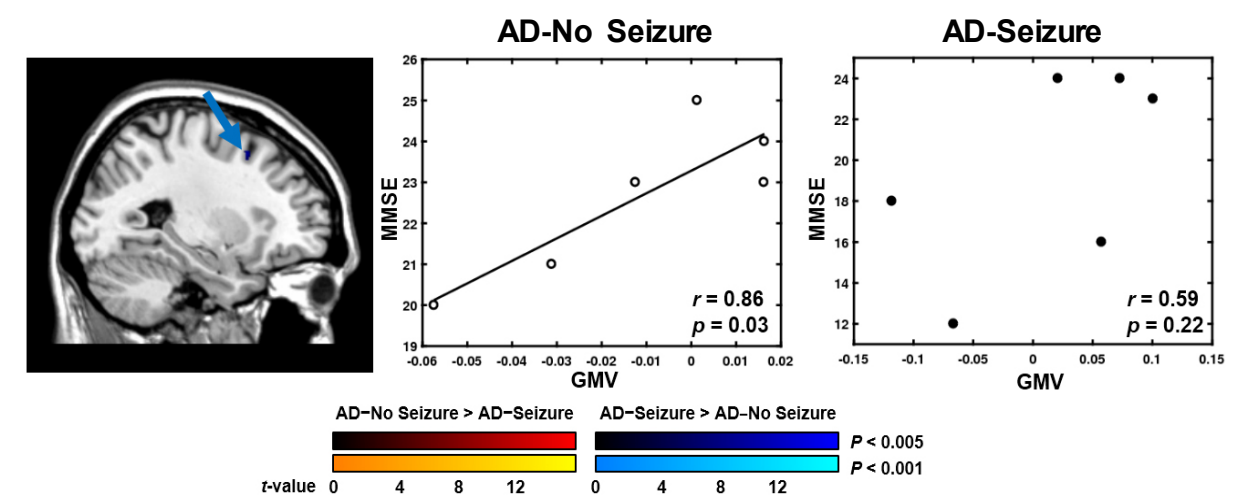

Figure 2. Correlation between MMSE score and gray matter volume of the region showing greater volume in patients with Alzheimer's disease (AD) who experience seizures than those who did not $(P<0.001$ and $P<0.005$, cluster size $\geq 5$, uncorrected for multiple comparisons). An arrow in a figure indicated a peak voxel served to perform the correlation analysis. MMSE, mini-mental state examination; AD-No Seizure, AD patients without seizures; AD-Seizure, AD patients with seizures; GMV, gray matter volume.

\section{Discussion}

The present study investigated cortical volumetric and cognitive function alterations related to the comorbidity of $A D$ and seizures. Comparison of $A D$ patients with seizures and those without seizures revealed seizure-related cortical alterations in the $A D$ brain in the form of either smaller or larger cortical volume in distributed cortical regions. Follow-up correlation analyses revealed that smaller volume of temporal regions in $A D$ patients with seizures was positively associated with cognitive function in the parahippocampal, middle temporal, and angular gyri. These temporal regions, which are well-known for their involvement in encoding and retrieval of information, overlap with regions that exhibited epileptiform activities in the AD-Seizure group. Therefore, our results suggest that smaller volume in temporal brain regions in these patients might indicate a synergistic effect of $A D$ and seizures that alters cortical volume and cognitive function.

By comparing cortical volumes between $A D$ patients with and without seizures, we have revealed cortical alterations in several regions that are specific to $A D$ patients with comorbid seizures. First, we found reduced cortical volume in specific structures in the AD-Seizure group. As the primary difference between groups is the presence of seizures, this may reflect seizure-related cortical volume loss. This result is in line with a previous study using an $A D$ mouse model which suggested that abnormal hyperexcitability of neurons can result in cortical volume loss 8 . The cortical volume alterations may also reflect cortical network reorganization via a synergistic effect of seizures and toxic accumulation of $A \beta$ in the $A D$ brain 6,8 . Second, we observed larger cortical volume in different regions in the
AD-Seizure group. The present study found both seizure-related cortical volumetric alterations. These findings are in accordance with a rat study reporting that abnormal electrophysiologic activity (i.e., Status Epilepticus and subsequent seizures) can trigger a reorganization of brain morphology by increasing cell size and decreasing dendrite arborization and spine density. ${ }^{17}$

To further investigate the relationship between cortical alteration and cognitive function disruption, we performed a correlation analysis. We hypothesized that, in the AD-Seizure group, regions with smaller cortical volume would be related to cognitive dysfunction. As expected, smaller cortical volume of the right parahippocampal, left middle temporal, and angular gyri predicted a low level of cognitive function in these patients. Those regions overlapped with regions shown in this patient group to display abnormal brain activity as assessed by EEG. They are also regions that are known for their contributions to memory function. ${ }^{18,19}$ Specifically, the right parahippocampal gyrus has been reported to be involved in encoding and maintenance of information. ${ }^{18}$ Additionally, the peak voxels in the left angular gyrus and middle temporal gyrus fell into BA 39 , which has been suggested to play a role in the retrieval of context-dependent semantic information. ${ }^{19}$ Taken together, these findings suggest that seizure-related cortical volume loss in temporal regions may contribute to cognitive dysfunction in the $A D$ brain.

Although the AD-No Seizure group was included in this study as a control group, and therefore was not of interest in the correlation analysis, we observed an association between cortical volume and cognitive function in this group. Volume of the right inferior frontal gyrus and cerebellum in the AD-No Seizure group was negatively correlated with cognitive function. There is literature that reports no sig- 
nificant positive correlation between MMSE score and cortical thickness of the right inferior frontal gyrus. ${ }^{20}$ However, as this study reported only significantly positive correlation coefficients, we cannot be sure that there were no negative or negligible positive correlations between cortical thickness of this region and cognition. Structural connectivity may, however, provide a possible explanation for the negative correlation seen between cortical volume or thickness and cognitive function. It has been recently reported that diffusion tractography revealed a structural connection between the right inferior frontal gyrus and the pre-supplementary motor area, ${ }^{21} \mathrm{a}$ region that has been noted to show relatively minimal cortical atrophy in the early stage of $A D .^{22}$ Based on these findings, we posit that the relatively preserved cortical volume of pre-supplementary motor regions may serve to preserve a morphometry of the right inferior frontal gyrus, and that this process is anti-correlated with cognitive functional decline. In contrast to several previous studies that have reported a positive correlation between cerebellar volume and MMSE scores in AD patients, ${ }^{23,24}$ we found a negative correlation between the left cerebellar region of the AD-No Seizure group, and cognitive function. Previous studies examined the right hemispheric cerebellum, which have been reported to be related to verbal function. The present study, however, found a negative correlation with the left hemispheric cerebellum, which is typically associated with non-verbal function. ${ }^{25,26}$ This may explain the discrepancies between the previous and present findings. In addition, the AD-No Seizure group also showed a positive correlation between the left middle frontal gyrus (BA 8) and cognitive function. This result is in line with a previous study ${ }^{20}$ that reported a significant association between GMV of the caudal part of the middle frontal gyrus and MMSE scores in AD patients. Although the correlation did not reach significance in our study, a marginally strong positive relationship was observed ( $r=0.59, p=0.22$ ).

The present study revealed significant differences in cortical volume between AD-Seizure and AD-No Seizure groups in distributed cortical regions including temporal, frontal, occipital, and cerebellar regions. To our knowledge, this is the first study to present these regional level cortical alterations in patients with $A D$ and comorbid seizures. Pathological alterations in the AD brain in the presence of seizures have been demonstrated mostly at the cellular micro-circuit level of hippocampal formation. ${ }^{8}$ Our findings provide a new macro-circuit level perspective of this phenomenon. These macro-scale cortical alterations have been suggested by Noebels ${ }^{8}$ in his review article. By examining neuroimaging findings in both $A D$, and tempo- ral lobe epilepsy, Noebels ${ }^{8}$ found that these two disorders showed extensive spatial overlap in volumetric atrophy and hypometabolism in the brain. This suggests that the common features in AD and temporal lobe epilepsy could be reflected in a cortical-level observation. In this regard, we believe that the present study provides evidence of cortical-level pathological change in the comorbidity of $A D$ and epilepsy.

One of the limitations of the present study is that the mean ages for each group were significantly different $(p<0.005$, Table 1$)$. As age is a crucial factor affecting cortical volume loss, ${ }^{27}$ we controlled for age by including it as a nuisance variable in group analyses. Another limitation of the study is the presence of group differences in cerebrovascular risk factors. Cerebrovascular risk factors such as hypertension and diabetes are known to have an adverse effect on cortical volume and thickness. ${ }^{28,29}$ These risk factors were not controlled across study groups: all the patients of the AD-Seizure group have one or more than one cerebrovascular risk factors such as hypertension, diabetes, and hyperlipidemia, whilst AD-No Seizure group have only one patient with hyperlipidemia. This difference might be related to the age difference between groups in that cerebrovascular risk factors are related to aging. ${ }^{30}$ Despite this limitation, both groups did not show much difference in the brain MRI examination: both groups had patients with diffuse brain atrophy and/or cerebral small vessel disease. In addition, angiography did not show any abnormality, hence it does not seem to be possible that vascular factors affect focal brain regions as shown in our findings. To rule out any possible confounding factors for cortical volumes, a future study needs age-matched and cerebrovascular risk factor-controlled/matched groups. In addition, the present study could not include healthy controls in terms of memory function, resulting in an absence of comparisons between patients and healthy controls. Since differences from the healthy controls were unknown for each patient samples in the present study, there was a limit to interpret the results. In a future study, a comparison with healthy controls also needs to be included to derive more convincing results. Lastly, the present study set a liberal alpha level without multiple comparisons corrections to find statistical results. Despite a possibility of increasing false positive rates, we used this liberal alpha level as a pilot study, which needs to observe a trend of cortical alterations in the $A D$ with seizure patients. Besides, with a small number of observations, it was hard to find any significant voxels after family-wise error correction. Considering the statistical power depends on the number of observations, the number of subjects should be increased for providing a statistically con- 
vincing evidence in the future study.

\section{Acknowledgments}

This study was supported by funding from Hanmi Pharmaceutical Co., Ltd. and by the National Research Foundation of Korea Grant from the Korean Government (NRF-2017R1A6A3A11036413). The funders had no role in study design, data collection, or analysis. The authors declare no competing financial interests.

\section{References}

1. Volicer L, Smith S, Volicer BJ. Effect of seizures on progression of dementia of the Alzheimer type. Dementia 1995;6:258-63.

2. Lott IT, Doran E, Nguyen VQ, Tournay A, Movsesyan N, Gillen DL. Down syndrome and dementia: seizures and cognitive decline. $J$ Alzheimers Dis 2012;29:177-85.

3. Vossel KA, Beagle AJ, Rabinovici GD, et al. Seizures and epileptiform activity in the early stages of alzheimer disease. JAMA Neurol 2013:70:1158-66.

4. Amatniek JC, Hauser WA, DelCastillo-Castaneda C, et al. Incidence and predictors of seizures in patients with Alzheimer's disease. Epilepsia 2006:47:867-72.

5. Scarmeas N, Honig LS, Choi $H$, et al. Seizures in Alzheimer disease: who, when, and how common? Arch Neurol 2009;66:992-7.

6. Palop JJ, Mucke L. Epilepsy and cognitive impairments in Alzheimer disease. Arch Neurol 2009;66:435-40.

7. Born HA. Seizures in Alzheimer's disease. Neuroscience 2015;286:251-63.

8. Noebels J. A perfect storm: converging paths of epilepsy and Alzheimer's dementia intersect in the hippocampal formation. Epilepsia 2011;52(Suppl 1):39-46

9. Velez-Pardo C, Arellano II, Cardona-Gomez P, Jimenez Del Rio M, Lopera F, De Felipe J. CA1 hippocampal neuronal loss in familial Alzheimer's disease presenilin-1 E280A mutation is related to epilepsy. Epilepsia 2004;45:751-6.

10. Palop JJ, Chin J, Roberson ED, et al. Aberrant excitatory neuronal activity and compensatory remodeling of inhibitory hippocampal circuits in mouse models of Alzheimer's disease. Neuron 2007;55:697-711.

11. McKhann GM, Knopman DS, Chertkow $H$, et al. The diagnosis of dementia due to Alzheimer's disease: recommendations from the National Institute on Aging-Alzheimer's Association workgroups on diagnostic guidelines for Alzheimer's disease. Alzheimers Dement 2011;7:263-9.

12. Lüders $H$, Acharya J, Baumgartner $C$, et al. Semiological seizure dassification. Epilepsia 1998:39:1006-13.

13. Ashburner J. A fast diffeomorphic image registration algorithm. Neurolmage 2007;38:95-113.

14. Lancaster JL, Tordesillas-Gutiérrez $\mathrm{D}$, Martinez $\mathrm{M}$, et al. Bias between
MNI and Talairach coordinates analyzed using the ICBM-152 brain template. Hum Brain Mapp 2007;28:1194-205.

15. Lancaster JL, Woldorff MG, Parsons LM, et al. Automated Talairach atlas labels for functional brain mapping. Hum Brain Mapp 2000;10:120-31.

16. Lancaster JL, Rainey $\mathrm{LH}$, Summerlin JL, et al. Automated labeling of the human brain: a preliminary report on the development and evaluation of a forward-transform method. Hum Brain Mapp 1997;5:238-42.

17. Luck D, Danion JM, Marrer C, Pham BT, Gounot D, Foucher J. The right parahippocampal gyrus contributes to the formation and maintenance of bound information in working memory. Brain Cogn 2010; 72:255-63.

18. Davey J, Thompson HE, Hallam G, et al. Exploring the role of the posterior middle temporal gyrus in semantic cognition: Integration of anterior temporal lobe with executive processes. Neuroimage 2016;137:165-77.

19. Baldaçara L, Borgio JGF, Moraes WA, et al. Cerebellar volume in patients with dementia. Rev Bras Psiquiatr 2011;33:122-9.

20. Thomann PA, Schläfer C, Seidl U, Santos VD, Essig M, Schröder J. The cerebellum in mild cognitive impairment and Alzheimer's disease - A structural MRI study. J Psychiatr Res 2008;42:1198-202.

21. Gottwald B, Wilde B, Mihajlovic Z, Mehdorn HM. Evidence for distinct cognitive deficits after focal cerebellar lesions. J Neurol Neurosurg Psychiatry 2004;75:1524-31.

22. Hokkanen LS, Kauranen V, Roine RO, Salonen O, Kotila M. Subtle cognitive deficits after cerebellar infarcts. Eur J Neuro/ 2006;13:161-70.

23. Colciaghi F, Finardi A, Nobili P, Locatelli D, Spigolon G, Battaglia GS. Progressive brain damage, synaptic reorganization and NMDA activation in a model of epileptogenic cortical dysplasia. PLoS One 2014;9:e89898.

24. Fjell AM, Amlien IK, Westlye LT, Walhovd KB. Mini-mental state examination is sensitive to brain atrophy in Alzheimer's disease. Dement Geriatr Cogn Disord 2009;28:252-8.

25. Swann NC, Cai W, Conner CR, et al. Roles for the pre-supplementary motor area and the right inferior frontal gyrus in stopping action: electrophysiological responses and functional and structural connectivity. Neurolmage 2012;59:2860-70.

26. Jacobsen JH, Stelzer J, Fritz TH, Chételat G, La Joie R, Turner R. Why musical memory can be preserved in advanced Alzheimer's disease. Brain 2015;138(Pt 8):2438-50.

27. Lemaitre $H$, Goldman AL, Sambataro F, et al. Normal age-related brain morphometric changes: nonuniformity across cortical thickness, surface area and gray matter volume? Neurobiol Aging 2012;33:617.e1-9.

28. Cardenas VA, Reed B, Chao $L L$, et al. Associations among vascular risk factors, carotid atherosclerosis, and cortical volume and thickness in older adults. Stroke 2012;43:2865-70.

29. Krishnadas R, McLean J, Batty GD, et al. Cardio-metabolic risk factors and cortical thickness in a neurologically healthy male population: Results from the psychological, social and biological determinants of ill health (pSoBid) study. Neurolmage Clin 2013;2:646-57.

30. Popa-Wagner A, Buga AM, Turner RC, Rosen CL, Toescu E. Cerebrovascular disorders: role of aging. J Aging Res 2012;2012:128146. 